\title{
Pandemia do novo Coronavírus e o funcionamento dos serviços odontológicos no Brasil: um seguimento de quatro meses
}

New Coronavirus pandemic and the functioning of dental services in Brazil: a four-month followup

Nuevo coronavirus pandemia y el funcionamiento de los servicios dentales en Brasil: un período de cuatro meses de seguimiento

\section{Resumo}

Objetivo: O presente estudo analisou o fechamento e abertura dos serviços odontológicos eletivos em resposta à Covid-19 e média móvel da curva epidêmica de quatro estados brasileiros, Amazonas, Ceará, São Paulo, Santa Catarina e Distrito Federal, além do posicionamento do Conselho Federal e Regionais de Odontologia. Método: Este foi um estudo de cinco casos a partir da análise de dados secundários e documental de publicações do Conselho Federal e Regionais de Odontologia com seguimento de março a junho de 2020. Calculou-se a média móvel de casos novos e óbitos diários por Covid-19 por estado. Resultados: A suspensão dos serviços ocorreu na segunda quinzena de março em todos os estados. A reabertura dos serviços odontológicos eletivos não obedeceu a nenhum critério de tendência da epidemia, tendo variado nos estados estudados entre abril e junho. Conclusão: A reabertura dos serviços não obedeceu a nenhum critério de tendência da curva epidemiológica do número de casos e óbitos e da taxa de ocupação de leitos de UTI, e não houve clareza dos documentos estaduais sobre mecanismos de fiscalização e multa para serviços que descumpriram as normas, aspectos que comprometem a segurança de profissionais e usuários dos serviços, especialmente pelo uso de aerossóis.

Palavras-chave: Coronavírus; Assistência odontológica; Política de saúde. 


\begin{abstract}
Objective: The present study analyzed the closure and opening of elective dental services in response to Covid-19 and moving average of the epidemic curve of four Brazilian states, Amazonas, Ceará, São Paulo, Santa Catarina and Federal District, in addition to the position of the Federal and Regional Council of Dentistry. Method: This was a study of five cases based on the analysis of secondary and documentary data from publications of the Federal and Regional Council of Dentistry with follow-up from March to June 2020. The mobile average of new cases and daily deaths by Covid-19 per state was calculated. Results: The suspension of services occurred in the second half of March in all states. The reopening of elective dental services did not comply with any epidemic trend criteria, and varied in the states studied between April and June. Conclusion: The reopening of the services did not comply with any criteria of trend of the epidemiological curve of the number of cases and deaths and the occupancy rate of ICU beds, and there is no clarity of state documents on enforcement mechanisms and fine for services that fail to meet the standards, aspects that compromise the safety of professionals and users of services, especially by the use of aerosols.
\end{abstract}

Keywords: Coronavirus; Dental care; Health policy.

\title{
Resumen
}

Objetivo: Este estudio analizó el cierre y apertura de servicios odontológicos electivos en respuesta a Covid-19 y promedio móvil de la curva epidémica en cuatro estados brasileños, Amazonas, Ceará, São Paulo, Santa Catarina y Distrito Federal, además del posicionamiento del Consejo Federal y Consultorios Odontológicos. Método: Se realizó un estudio de cinco casos basado en el análisis de datos secundarios y documentales de publicaciones del Consejo Federal y Regional de Odontología con un seguimiento de marzo a junio de 2020. Se calculó el promedio móvil de nuevos casos y muertes diarias. por Covid -19 por estado. Resultados: La suspensión de servicios ocurrió en la segunda quincena de marzo en todos los estados. La reapertura de los servicios odontológicos electivos no siguió ninguna tendencia de la epidemia, habiendo variado en los estados estudiados entre abril y junio. Conclusión: La reapertura de los servicios no siguió ningún criterio tendencial de la curva epidemiológica del número de casos y muertes y la tasa de ocupación de camas de UCI, y no hay claridad en los documentos estatales sobre los mecanismos de inspección y multas por los servicios que no cumplen. con los estándares, aspectos que comprometen la seguridad de los profesionales y usuarios de los servicios, especialmente por el uso de aerosoles.

Palabras clave: Coronavirus; Atención odontológica; Política de salud.

\section{Introdução}

O ano de 2020 foi marcado pela pandemia de Covid-19, causada pelo novo coronavírus SARS-CoV-2, detectado pela primeira vez no dia 31 de dezembro de 2019, na cidade de Wuhan, China (Lana et al., 2020). Em janeiro de 2020, a Organização Mundial de Saúde (OMS) declarou a epidemia como emergência internacional, e no dia onze de março de 2020 oficializou a situação de pandemia (OMS \& OPAS, 2020).

Já no final do mês de abril de 2020, a Europa apresentava quase metade de todos os casos registrados no mundo, sendo os países com maior número de casos confirmados Espanha, Itália, Reino Unido, Alemanha e França, enquanto o Brasil ocupava a $10^{\text {a }}$ posição mundial (Pereira, 2020). Em dois meses, o epicentro da pandemia passou para as Américas, com destaque para os Estados Unidos (EUA) e Brasil, respectivamente em primeiro e segundo lugar, e incluiu outros países como Peru, Chile e México, assim como Índia e África do Sul (Pereira, 2020). Nesse sentido, diversos países decretaram a suspensão de serviços considerados não essenciais, incluindo os atendimentos odontológicos eletivos ou de rotina.

Em março de 2020, a Associação Dentária Americana (ADA) recomendou que cirurgiões-dentistas dos EUA adiassem procedimentos eletivos, pelo alto risco de contágio com uso de aerossóis na prática clínica (ADA, 2020). No Reino Unido, no mesmo período, o Chief Dental Officer também anunciou a interrupção imediata de atendimentos não urgentes (Grossman et al., 2020). Na Inglaterra, um mês após a suspensão dos atendimentos odontológicos de rotina, a demanda por atendimentos de urgência aumentou, sendo as queixas dentárias $80 \%$ das ligações não relacionadas à Covid-19 recebidas pelo NHS/Londres (Grossman et al., 2020). Na Argentina, no Chile e Brasil a suspensão de atendimentos eletivos também ocorreu (Carrer et al., 2020; Santos-Lopez \& Catrian-Fernandez, 2020), sendo que no Brasil o Ministério da Saúde (MS) suspendeu vários serviços eletivos no Sistema Único de Saúde (SUS), incluindo consultas e procedimentos odontológicos.

A Coordenação Geral de Saúde Bucal orientou a manutenção apenas dos atendimentos de urgência, e, participação 
das equipes de saúde bucal da atenção básica nas ações do "FAST-TRACK COVID-19", como triagem, classificação dos usuários e organização dos serviços (Brasil, 2020a). Em março de 2020, o Conselho Federal de Odontologia (CFO) solicitou ao MS a suspensão dos atendimentos eletivos na rede pública. Para os serviços da rede privada, a entidade solicitou maior rig or no controle de protocolos de prevenção e desinfecção para evitar a transmissão (CFO, 2020). Entretanto, verificou-se que a abertura e fechamento desses serviços nas diferentes regiões do país acompanharam as decisões dos governos estaduais e municipais.

Neste sentido, o presente estudo analisou o fechamento e abertura dos serviços odontológicos eletivos e de rotina, considerando a média móvel e a tendência da curva epidêmica de cinco estados brasileiros, no período de fevereiro a junho de 2020. Assim, contribui para identificar a resposta estadual e federal no Brasil frente à pandemia no contexto da adoção de medidas relacionadas ao fechamento e abertura dos serviços odontológicos e sua possível relação com a média móvel e a tendência da curva epidêmica em estados brasileiros no referido período. Também foram analisados posicionamentos do CFO e dos Conselhos Regionais dos casos selecionados, enquanto entidade de classe reguladora do exercício profissional.

\section{Metodologia}

Estudo de cinco casos de estados brasileiros em resposta à Covid-19 na área odontológica a partir da análise de dados secundários e documental de publicações do Conselho Federal de Odontologia (CFO) e dos Conselhos Regionais de Odontologia (CRO) dos estados do Amazonas (AM), Ceará (CE), São Paulo (SP), Santa Catarina (SC) e do Distrito Federal (DF), no período compreendido entre fevereiro/março a junho de 2020, um follow-up de quatro meses em cada estado.

O CFO foi incluído por ser a entidade nacional, reguladora do exercício profissional. Entre os estados, foram analisados os primeiros que apresentaram o maior número de casos e crescimento da epidemia (clusters), sendo estes SP, CE e AM. Por ser o primeiro estado a reabrir as atividades comerciais, incluiu-se o estado de SC, e o DF, por ser a capital, contemplando na amostra um estado de cada região do país.

As informações referentes ao número de casos e de óbitos pelo Covid-19, bem como taxa de ocupação de leitos foram coletadas preferencialmente nos sites oficiais das Secretarias Estaduais de Saúde (SES) dos cinco estados, as quais foram complementadas e cruzadas com informações disponíveis pelo Ministério da Saúde (https://covid.saude.gov.br/), pelo Conselho Nacional dos Secretários de Saúde (http://www.conass.org.br/painelconasscovid19/), e ainda em publicações nos sites de jornais de grande circulação nacional (O Globo, Folha de São Paulo e Estadão) e de jornais estaduais. Foram coletados também indicadores demográficos e socioeconômicos de cada estado no Instituto Brasileiro de Geografia e Estatística (IBGE). Realizada análise através do software Microsoft Excel (2016) e produção de média móvel de casos novos e dos óbitos diários por estado, com intervalo de 14 dias. E analisado a tendência da curva epidêmica no dia da abertura dos serviços odontológicos considerando-a como ascendente, descendente ou estável na análise visual da tendência da média móvel.

As datas e informações sobre fechamento e reabertura dos serviços foram confirmadas nos sites oficiais das SES, e os posicionamentos do CFO e respectivos Conselhos Regionais foram analisados a partir das publicações oficiais das autarquias. O período de coleta ocorreu de fevereiro/março a 30 junho de 2020, follow up de 4 meses iniciado a partir do primeiro caso registrado de Covid-19 no país. As notícias e produções do CFO republicadas pelos Conselhos Regionais não foram incluídas nas análises regionais. Para análise dos posicionamentos do CFO e Conselhos Regionais foi utilizado o software de análise qualitativa de dados NVivo-11.

\section{Resultados e Discussão}

A suspensão dos serviços odontológicos ocorreu na segunda quinzena de março de 2020 em todos os estados 
estudados. Já a abertura dos serviços eletivos ou de rotina não obedeceu a nenhum critério claro de tendência da epidemia, tendo variado entre os estados. O tempo decorrido entre o primeiro caso e a suspensão do serviço odontológico variou de 4 dias no Ceará (CE) até 26 dias no estado de São Paulo (SP) (Tabela 1). Quanto ao tempo em que os serviços ficaram fechados variou de 11 dias apenas no Distrito Federal (DF) a 73 dias no CE. Ou seja, o Ceará foi o estado que instituiu de forma mais rápida a suspensão dos serviços odontológicos eletivos e o que permaneceu mais tempo com os serviços suspensos. As informações epidemiológicas no dia da reabertura dos serviços eletivos foram distintas entre os estados e a tendência da curva epidemiológica de casos e óbitos apresentou-se como estável (DF e SC) e ascendente (AM, SP e CE) no dia da abertura dos serviços (Tabela 1, Figura 1 e 2). Isso pode ser explicado pelas repercussões locais e nacionais na expansão da pandemia, especialmente midiáticas naquele período.

Entre os casos selecionados, há diferenças importantes no Índice de Desenvolvimento Humano (IDH), taxa de ocupação e analfabetismo, sendo os melhores indicadores nos estados de SC, SP, DF, e os piores no AM e CE (Tabela 1).

Tabela 1. Principais eventos relacionados à pandemia e as respostas das entidades odontológicas e governamentais por data e número de casos acumulados de Covid-19 em cinco estados brasileiros, 2020.

\begin{tabular}{|c|c|c|c|c|c|}
\hline Indicadores por estado & Amazonas & Ceará & $\begin{array}{l}\text { Distrito } \\
\text { Federal }\end{array}$ & São Paulo & $\begin{array}{c}\text { Santa } \\
\text { Catarina }\end{array}$ \\
\hline \multicolumn{6}{|l|}{ Demográficos } \\
\hline População ${ }^{10}$ & 4.144 .597 & 9.132 .078 & 3.015 .268 & 45.919 .049 & 7.164.788 \\
\hline Densidade demográfica $\left(\mathrm{hab} / \mathrm{km}^{2}\right)^{10}$ & 2,66 & 61,33 & 523,41 & 184,99 & 74,84 \\
\hline$\%$ população urbana ${ }^{11}$ & 79,09 & 75,09 & 96,58 & 95,94 & 83,99 \\
\hline \multicolumn{6}{|l|}{ Socioeconômica } \\
\hline$\%$ de população 14 anos ou mais ocupada ${ }^{12}$ & 41,0 & 42,6 & 69,0 & 66,9 & 77,3 \\
\hline IDH ${ }^{13}$ p & 0,733 & 0,735 & 0,850 & 0,826 & 0,808 \\
\hline Taxa de analfabetismo da população de 15 anos ou mais ${ }^{12}$ & 5,4 & 13,6 & 2,7 & 2,6 & 2,3 \\
\hline \multicolumn{6}{|l|}{ Resposta à epidemia e sistema de saúde } \\
\hline Data do caso 1 & $15 / 03$ & $15 / 03$ & $05 / 03$ & $26 / 02$ & $25 / 02$ \\
\hline $\begin{array}{l}\text { Data de suspensão dos serviços odontológicos eletivos e de } \\
\text { rotina }\end{array}$ & $23 / 03$ & $19 / 03$ & $20 / 03$ & $23 / 03$ & $18 / 03$ \\
\hline $\begin{array}{l}\text { Tempo entre primeiro caso e suspensão dos serviços } \\
\text { odontológicos eletivos e de rotina }\end{array}$ & 8 dias & 4 dias & 15 dias & 26 dias & 22 dias \\
\hline $\begin{array}{l}\text { Data da reabertura dos serviços odontológicos eletivos e de } \\
\text { rotina }\end{array}$ & $25 / 05$ & $01 / 06$ & $01 / 04$ & $01 / 06$ & $06 / 04$ \\
\hline $\begin{array}{l}\text { Tempo em dias entre a data da suspensão e reabertura dos } \\
\text { serviços odontológicos eletivos e de rotina }\end{array}$ & 64 & 73 & 11 & 70 & 18 \\
\hline $\begin{array}{l}\text { Número de óbitos no dia da reabertura dos serviços } \\
\text { odontológicos eletivos e de rotina }\end{array}$ & 23 & 94 & 1 & 169 & 1 \\
\hline $\begin{array}{l}\text { Média móvel de casos no dia da reabertura dos serviços } \\
\text { odontológicos eletivos e de rotina (14 dias) }\end{array}$ & $1.240,2$ & $1.907,6$ & 24,0 & $3.445,0$ & 52,2 \\
\hline $\begin{array}{l}\text { Taxa de Ocupação dos leitos de UTI }(\%) \text { no dia da reabertura } \\
\text { dos serviços odontológicos eletivos e de rotina }\end{array}$ & $82,0^{14}$ & $87,8^{15}$ & $\mathrm{X}$ & $84,0^{16}$ & $\mathrm{X}$ \\
\hline $\begin{array}{l}\text { Notas conjuntas entre Conselhos Regionais e Secretarias } \\
\text { Estaduais (sim ou não) }\end{array}$ & Não & Não & Não & Sim & Sim \\
\hline $\begin{array}{l}\text { Perfil da curva epidemiológica de casos novos no dia da } \\
\text { reabertura dos serviços odontológicos eletivos e de rotina }\end{array}$ & Ascendente & Ascendente & Estável & Ascendente & Estável \\
\hline
\end{tabular}

$\mathrm{X}$ = informação não disponível

Fontes: ${ }^{10}$ IBGE (2020), ${ }^{11}$ Atlas Brasil (2020), ${ }^{12}$ IBGE (2019), ${ }^{13}$ Wikipédia (2017), ${ }^{14}$ Amazonas (2020a), ${ }^{15}$ Ceará (2020a), ${ }^{16}$ São Paulo (2020a).

SP, SC e DF apresentaram curva ascendente nos dois gráficos e o Amazonas apresentou um decréscimo tanto de casos novos quanto de óbitos no período. O CE apresentou ao final do período uma estabilização na média de casos novos e um decréscimo na média dos óbitos. Verificou-se nos cinco estados que a decisão de suspensão e reabertura dos serviços odontológicos foi realizada pelas autoridades sanitárias através de publicações emitidas pelas suas Secretarias Estaduais de Saúde (SES) e a abertura dos serviços odontológicos não esteve relacionada à redução de tendência de casos (Figuras 1 e 2); à 
Research, Society and Development, v. 10, n. 12, e200101220266, 2021

(CC BY 4.0) | ISSN 2525-3409 | DOI: http://dx.doi.org/10.33448/rsd-v10i12.20266

exceção do Ceará e do Amazonas que apontaram alguns aspectos técnicos e sanitários como a disponibilidade de leitos de UTI, taxa de transmissão e a ocorrência de novos casos.

Figura 1. Média móvel de casos novos de Covid-19 por estado, considerando um intervalo de 14 dias. Brasil, 2020.
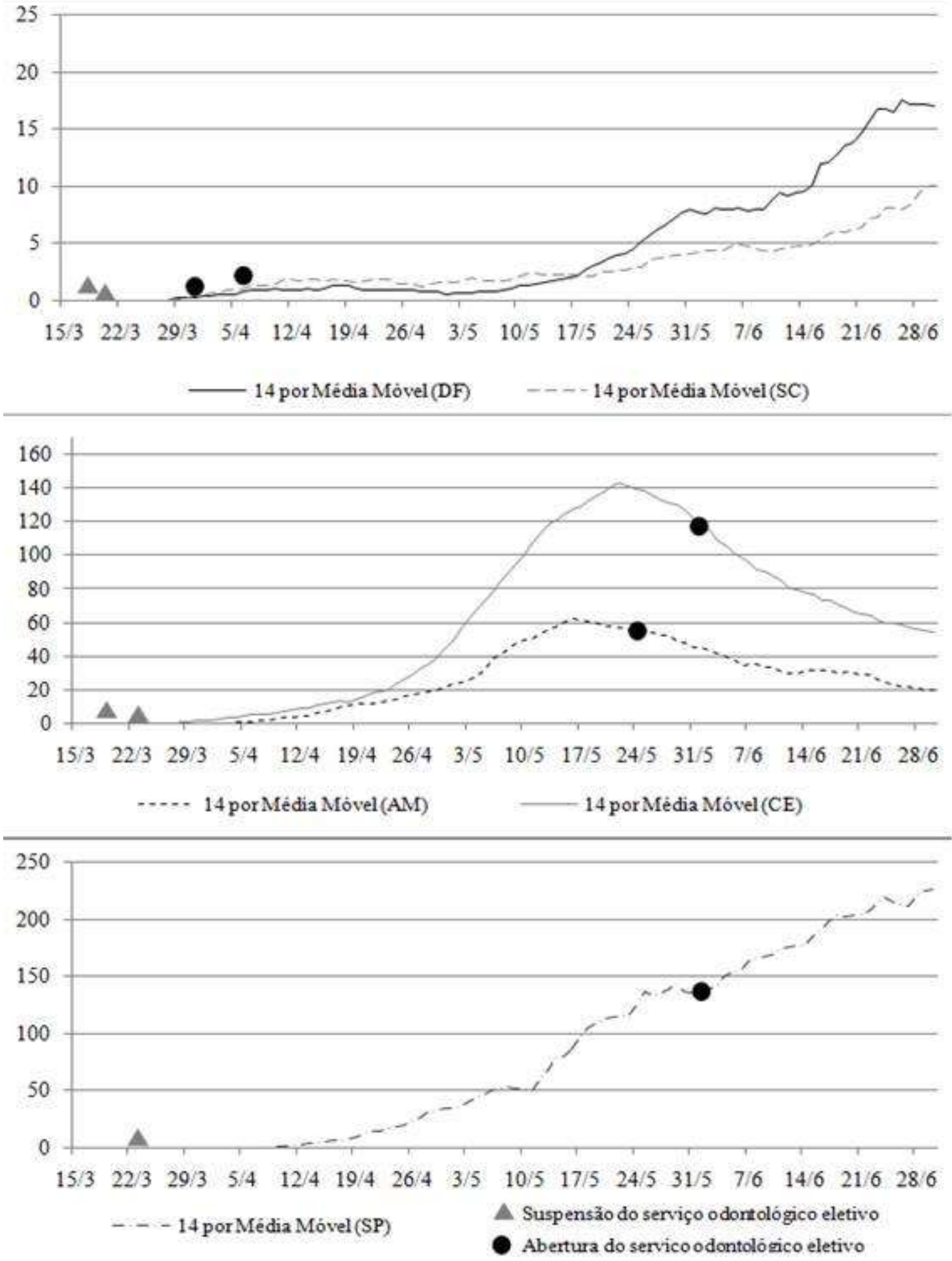

Fonte: Secretaria de Saúde do Distrito Federal, (2020); Governo de Santa Catarina, (2020); Fundação de Vigilância em Saúde do Amazonas, (2020); IntegraSUS Governo do Estado do Ceará, (2020); Fundação Sistema Estadual de Análise de Dados (São Paulo), (2020). 
Research, Society and Development, v. 10, n. 12, e200101220266, 2021

(CC BY 4.0) | ISSN 2525-3409 | DOI: http://dx.doi.org/10.33448/rsd-v10i12.20266

Figura 2. Média móvel dos óbitos diários de Covid-19 por estado, considerando um intervalo de 14 dias. Brasil, 2020.
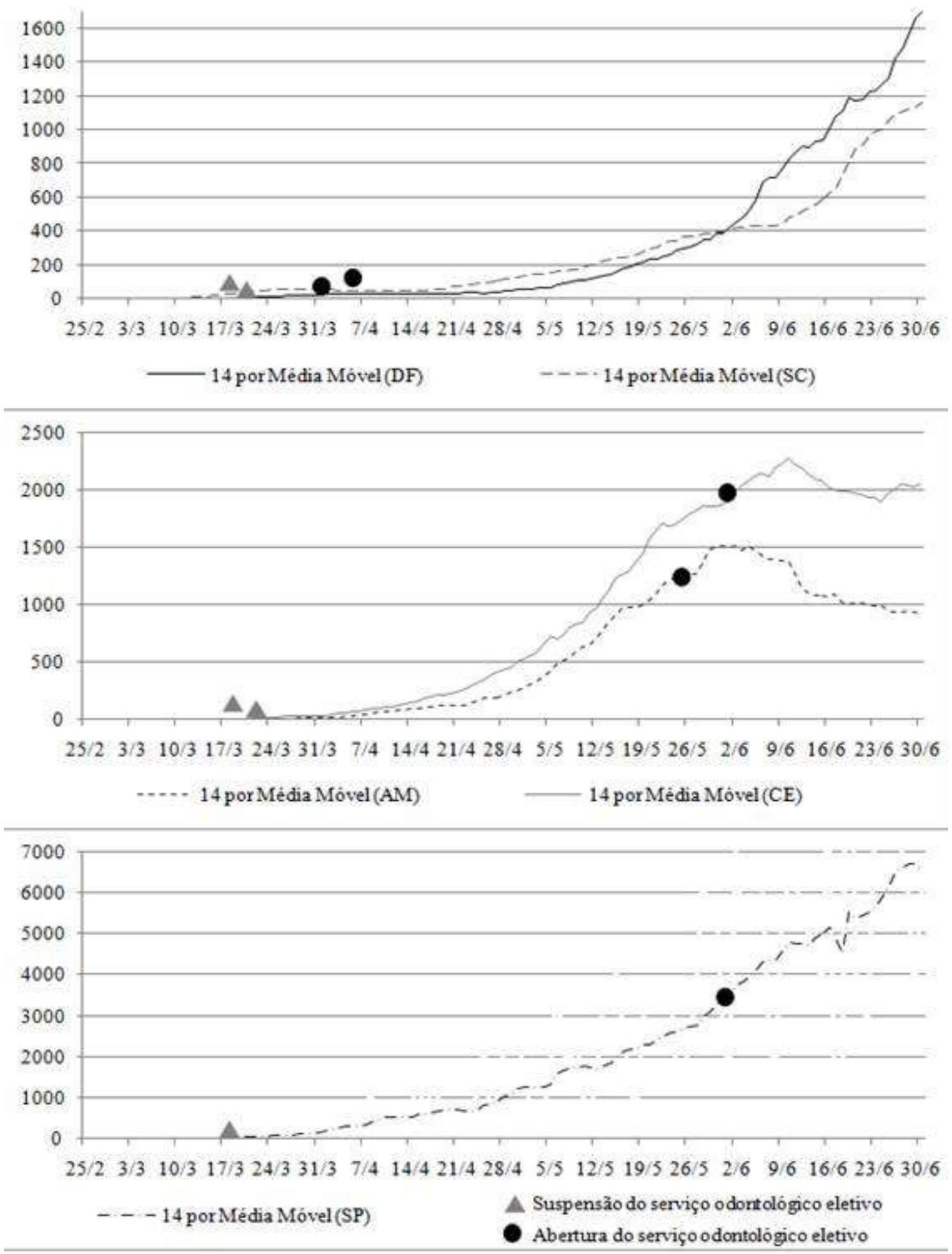

Fonte: Secretaria de Saúde do Distrito Federal, (2020); Governo de Santa Catarina, (2020); Fundação de Vigilância em Saúde do Amazonas, (2020); IntegraSUS Governo do Estado do Ceará, (2020); Fundação Sistema Estadual de Análise de Dados (São Paulo), (2020).

Santa Catarina confirmou seu primeiro caso em 25 de fevereiro de 2020 e vinte dias depois, o CRO-SC e a Vigilância Sanitária lançaram Nota Técnica conjunta recomendando que a odontologia nos serviços públicos, privados e clínicas em cursos de graduação e pós-graduação realizassem somente atendimentos de urgência (Santa Catarina, 2020). Em 6 abril, o governo estadual autorizou o funcionamento das atividades de diversos profissionais autônomos/liberais de saúde, incluindo os 
odontólogos (Santa Catarina, 2020). Nessa data, SC tinha 417 casos confirmados e 1 óbito nas últimas 24 horas (Tabela 1). No dia 27 de abril, a taxa de ocupação de leitos era de 16,8\% (Globo.com, 2020). Além das duas Notas Técnicas conjuntas, o CRO-SC repercutiu publicações da SES, e destacou protocolos de higienização, uso de equipamentos de proteção individual, limpeza e desinfecção de superfícies, entre outros assuntos (CRO-SC, 2020). No dia 20 de maio, o CRO-SC encaminhou ofício ao governador e à SES solicitando autorização para funcionamento de instituições de ensino (CRO-SC, 2020).

Tysiac-Mista \& Dziedzic (2020) defendem a importância de orientações dos setores governamentais e entidades representativas em um momento de crise na saúde, como é o caso da pandemia do Covid-19, demonstrando o impacto destas na tomada de decisão dos dentistas em manter ou não o funcionamento dos serviços odontológicos. No Brasil, o CFO em todo o período analisado, defendeu ajustes nos protocolos e aumento de procedimentos de biossegurança e a continuidade dos atendimentos odontológicos no setor privado (CFO, 2020).

No estado de São Paulo, o primeiro caso confirmado foi em 26 de fevereiro (São Paulo, 2020b). Em 13 março o CROSP publicou sobre cuidados e prevenção, quando SP já apresentava 56 casos confirmados (CRO-SP, 2020). Três dias após o registro das primeiras mortes no estado, a autarquia recomendou a realização dos atendimentos odontológicos apenas de urgência, atuando junto ao Conselho de Secretários Municipais de Saúde, no sentido de suspender a realização de atendimentos eletivos nos serviços públicos (São Paulo, 2020b). No dia 23 de março, o CRO-SP se posicionou em relação à suspensão dos atendimentos eletivos no setor público e privado, respaldado pelo documento do Grupo Técnico Odontológico da Divisão de Serviços de Saúde do Centro de Vigilância Sanitária da Secretaria Estadual de Saúde de SP (CRO-SP, 2020). Setenta dias após o anúncio oficial da suspensão dos atendimentos eletivos, o CRO-SP se manifestou a favor da abertura dos serviços odontológicos (São Paulo, 2020b; CRO-SP, 2020). Neste dia, o estado acumulava 111.296 casos e 7.667 mortes, com ocupação de $84 \%$ dos leitos de UTI do estado e perfil da curva epidemiológica de casos e óbitos ascendente (Tabela 1).

No Ceará, os três primeiros casos foram confirmados dia 15 de março e um dia depois o governo estadual decretou situação de emergência (Ceará, 2020b). Nessa mesma data, o CRO-CE publicou ofício, recomendando que fossem realizados apenas serviços de urgência e emergência nos atendimentos odontológicos públicos e privados (CRO-CE, 2020). No dia 19 março, o governo estadual vetou atividades odontológicas que não fossem de urgência e, logo em seguida, a Secretaria de Saúde do Estado publicou uma Nota Técnica com orientações para os serviços odontológicos da rede de atenção à saúde bucal (Ceará, 2020b). No dia 24 de março, os três primeiros óbitos no estado foram confirmados (CRO-CE, 2020). Em 6 de abril, eram 72 óbitos no estado, e o governo estadual manteve as medidas restritivas (Ceará, 2020b). Nessa mesma data, o CRO-CE enviou ao governo estadual uma proposta de normativa em apoio ao retorno dos atendimentos eletivos e tal solicitação foi reiterada no dia 30 de abril (Ceará, 2020b; CRO-CE, 2020). Em maio, o Conselho criou uma câmara técnica com diversos profissionais, professores universitários e com a Associação Brasileira de Odontologia do estado (ABO-CE) para elaboração de um manual orientador para o atendimento odontológico (CRO-CE, 2020).

No mês de junho, algumas atividades relativas à cadeia da construção civil e da saúde, dentre elas, as atividades odontológicas eletivas foram retomadas (Ceará, 2020b). No referido decreto, o governo defendeu a importância da retomada progressiva das atividades econômicas. A Secretaria Estadual de Saúde nesse mês publicou a Nota Técnica com orientações para o retorno dos serviços odontológicos em 3 fases, destacando que a decisão para a mudança de fase deveria ser tomada de forma regional com base na avaliação epidemiológica a cada oito dias (Ceará, 2020b). No dia 10 de junho, o CRO-CE publicou ofício com orientações referentes ao retorno dos serviços odontológicos no Ceará (CRO-CE, 2020). Na data de retorno das atividades, a taxa de ocupação dos leitos de UTI por Covid-19 era de 87,8\% (Tabela 1).

Ceará e São Paulo, estados que permaneceram mais tempo sem atendimento eletivo (73 e 70 dias, respectivamente), foram aqueles que mais sofreram pressão para reabertura pelos respectivos Conselhos Regionais (CRO-SP, 2020, CRO-CE, 2020). Nos demais, parece não ter sido necessário, haja vista menor tempo entre fechamento e reabertura. A provável 
explicação pode estar muito mais na cobrança realizada pelos profissionais aos órgãos de classe, do que na análise científica das curvas da epidemia. Além disso, observa-se que boa parte dos documentos estaduais são recomendações sem clareza dos mecanismos de controle, fiscalização e multa para serviços que as descumpram. Nesse aspecto, o CRO-SC reforçou em suas publicações que o descumprimento das recomendações estaduais e municipais, sobretudo em relação a biossegurança e uso de EPIs, era infração ética e deveria ser denunciada ao Conselho (CRO-SC, 2020).

O Distrito Federal registrou o primeiro caso de Covid-19 no dia 5 de março (Distrito Federal, 2020). No dia 19 de março, o governo suspendeu diversos serviços e atividades no Decreto $\mathrm{n}^{\circ} 40.539 / 2020$, mas não incluiu as clínicas odontológicas, o que levou o CRO-DF a solicitar esse fechamento (Distrito Federal, 2020; CRO-DF, 2020). Logo em seguida, o governador publicou novo decreto autorizando apenas atendimentos de urgência e emergência (Distrito Federal, 2020). Após 11 dias da suspensão dos atendimentos eletivos e de rotina, o governo do DF autorizou o funcionamento dos serviços odontológicos eletivos (Distrito Federal, 2020). Neste dia, o DF apresentou 1 óbito e 37 novos casos (Tabela 1). Mesmo com essa autorização, o CRO-DF manteve a recomendação à categoria de suspensão dos atendimentos eletivos (CRO-DF, 2020).

O Amazonas teve seu primeiro caso de Covid-19 confirmado em 15 de março, e no dia seguinte o CRO-AM propôs aos órgãos públicos e privados a suspensão do atendimento odontológico eletivo (Amazonas, 2020b; CRO-AM, 2020). Em 23 de março, oito dias após a confirmação do primeiro caso e com um total de 23 casos confirmados, o governo estadual decretou a suspensão de todos os atendimentos eletivos em clínicas e consultórios odontológicos públicos e privados (Amazonas, 2020b). Na mesma data, a Coordenação Estadual de Saúde Bucal emitiu Nota Técnica orientando os profissionais sobre o manejo odontológico frente a situação de pandemia (Amazonas, 2020b).

Após 63 dias de fechamento dos serviços eletivos, em 25 de maio, o governo estadual autoriza o retorno dos serviços odontológicos eletivos e de rotina (Amazonas, 2020b), e nesta data eram 476 casos novos, 25 óbitos confirmados nas últimas 24 horas e taxa de ocupação de leitos de $82 \%$ para UTI e 51\% para leitos clínicos (Tabela 1). Ainda neste dia, a Fundação de Vigilância à Saúde publicou um plano com recomendações a flexibilização gradual dos setores de comércio e serviços, que incluía os serviços odontológicos na fase 1, com data prevista para 01 junho e estabelecendo que a taxa de ocupação dos leitos deveria estar em 30\% (FVS-AM, 2020). No dia 28 de maio, o governo estadual publicou um decreto com o cronograma de reabertura das atividades econômicas, considerando os critérios de disponibilidade de leitos de UTI, taxa de transmissão e ocorrência de novos casos (Amazonas, 2020b). Este decreto estabeleceu o funcionamento dos serviços odontológicos da cidade de Manaus a partir de junho e nas demais cidades esta reabertura ficaria a critério do gestor municipal (Amazonas, 2020b). Em todo período analisado o CRO-AM repercutiu as publicações governamentais relacionadas ao funcionamento dos serviços odontológicos (CRO-AM, 2020).

Já o Conselho Federal de Odontologia (CFO), desde o início da pandemia, publicou orientações e alertas sobre a Covid-19 e sobre os procedimentos odontológicos. No dia 16 de março, o presidente do CFO solicitou ao Ministro da Saúde a suspensão das atividades odontológicas na rede pública nacional que não fossem de urgência e emergência, e recomendação aos serviços privados de saúde de ampliar o rigor e medidas de controle da biossegurança (CFO, 2020). Depois disso, não foram encontradas publicações sobre o posicionamento do CFO com relação à abertura dos serviços odontológicos nos estados brasileiros até o dia 30 de junho. Ainda assim, as normativas por ele publicadas foram republicadas por todos os conselhos estaduais analisados. O CFO e Conselhos Regionais também atuaram e se posicionaram em outros temas relacionados ao cenário da pandemia, com foco principal na produção e divulgação de materiais orientadores, educativos sobre biossegurança e em medidas de redução dos impactos financeiros negativos decorrentes da crise sanitária (CFO, 2020; CRO-SC, 2020; CROSP, 2020; CRO-DF, 2020; CRO-AM, 2020; CRO-CE, 2020).

Entre as medidas financeiras destacam-se o envio de demandas por parte dos Conselhos Regionais aos municípios e estado solicitando redução nos tributos incidentes sobre a odontologia, prorrogação de prazos de pagamento de tributos e apoio 
na concessão de linhas de crédito específicas junto aos bancos públicos (CRO-SC, 2020; CRO-SP, 2020; CRO-DF, 2020; CRO-AM, 2020; CRO-CE, 2020). O CRO-SP, especialmente, enviou também pedido de reparação junto à Agência Nacional de Saúde Suplementar (ANS) para os prestadores de serviços de saúde bucal, além de se manifestar pelo não congelamento dos salários dos profissionais da odontologia que atuam na rede pública (CRO-SP, 2020). A autarquia destacou a dificuldade de obtenção dos equipamentos de proteção individual (EPIs) no mercado, principalmente de máscaras e jalecos descartáveis enviando comunicação à Associação Brasileira da Indústria de Artigos e Equipamentos Médicos, Odontológicos, Hospitalares e de Laboratórios (ABIMO), no sentido atenção especial à demanda dos EPIs (CRO-SP, 2020).

Na questão econômica, o CFO prorrogou o pagamento da anuidade de 2020, destacou a sua solicitação ao Ministro da Economia de medidas que beneficiassem os profissionais da odontologia, defendeu a suspensão por tempo indeterminado da cobrança do Fies aos estudantes universitários, solicitou à ANS medidas protetivas aos cirurgiões-dentistas prestadores de serviços em planos odontológicos, defendeu aprovação do Projeto de Lei n ${ }^{\circ}$ 2424/2020 para concessão de linha de crédito para profissionais liberais, que atuem como pessoa física, publicou a Resolução 226/2020, que regulamenta o exercício da odontologia a distância no período de pandemia (CFO, 2020).

Chama a atenção que, contrariamente ao setor privado, no âmbito do serviço público, a publicação do Ministério da Saúde (MS) que orienta os atendimentos no SUS (Nota Técnica 16/2020 de 17/06/20), mantém a realização apenas de atendimentos das urgências odontológicas nos estabelecimentos da Atenção Primária à Saúde, colaboração dos profissionais nas ações do Fast Track Covid-19, orientação à população e uso, quando possível, de contato por telefone ou outros meios digitais com usuários da área adstrita (Brasil, 2020b). E, apesar de no dia 23 de maio de 2020 uma Nota Informativa recomendar que os cirurgiões-dentistas do SUS realizassem exames para diagnóstico de Covid-19, através da "coleta de swab" (Brasil, 2020c), não foi encontrada referência a esse documento nos sites das Secretarias Estaduais de Saúde e Conselhos Regionais dos casos analisados. No documento, o MS faculta essa decisão aos gestores considerando a necessidade de capacitação e EPIs adequados (Brasil, 2020c).

Para os serviços do Centro de Especialidade Odontológica (CEO) e Laboratório Regional de Prótese Dentária, o MS também ressalta o papel do gestor local para decisão sobre o funcionamento dos serviços, baseado nas características epidemiológicas locais (Brasil, 2020b). O MS recomendou o uso do Guia Interino desenvolvido pela Universidade Federal de Pelotas, o qual apresenta uma adaptação das recomendações da American Dental Association (ADA) (Brasil, 2020b). A inserção do cirurgião-dentista em comissão interdisciplinar para enfrentamento da pandemia contribuiu para produção e atualização dos protocolos para as equipes de saúde bucal, com estabelecimento de critérios para eleger as demandas urgentes e treinamento das equipes para aplicação das novas medidas de biossegurança (Almeida et al. 2020).

Entre 2019 e 2020, houve aumento do número de equipes de saúde bucal em 25 estados e da cobertura em 17 estados, entretanto, comparando-se o primeiro quadrimestre desses dois anos, verificou-se redução significativa no número e cobertura das primeiras consultas odontológicas programáticas e de atendimentos devido a abscesso dento-alveolar e dor de dente (Lucena et al., 2020). Pode-se atribuir essa redução à pandemia tendo em vista a suspensão dos atendimentos eletivos no SUS desde março, diferentes formas de organização dos atendimentos de urgência, bem como de menor acesso e provimento dos EPIs e à própria mudança de comportamento dos usuários na busca pelos serviços (Lucena et al., 2020). Também foi observada uma redução de 65,6\% na média de consultas clínicas odontológicas no setor público no Brasil, representando mais de 21 mil consultas clínicas, comparando o período de 2015 a 2019 com o ano de 2020 (Marques et al., 2020). Com relação às consultas e procedimentos de urgência, a redução foi de $42,5 \%$ em serviços de atenção primária e de 44,1\% na atenção especializada, entre 2020 e 2019 (Cunha et al., 2021). No setor privado, além da redução de atendimentos, a exigência de novos protocolos mais rígidos de biossegurança aumentou a demanda de consumo de EPIs e dos custos operacionais (Nunes et al., 2020). Com base nos novos protocolos de biossegurança, estima-se que os custos de uma única consulta odontológica 
aumentaram 19,05 vezes e o custo anual com assistência à saúde bucal aumentou 9,5 vezes, no Brasil (Cavalcanti et al., 2020).

O cenário pandêmico tem gerado impactos nos serviços odontológicos e na saúde bucal em todo o mundo, dentre os quais redução do número de pacientes, aumento dos custos do tratamento, do tempo de espera, do número de atendimentos de urgência e de extrações dentárias, maior risco de saúde ocupacional, além do aumento de demandas por teleatendimento. No Reino Unido, estudo apontou que 71,5\% dos consultórios só poderiam sustentar suas finanças comerciais por apenas 3 meses ou menos (Barabari \& Moharamzadeh, 2020).

Na Polônia, houve uma diminuição significativa no número de pessoas atendidas pelos serviços odontológicos públicos e privados, de 47,1\% para 3,0\% (Tysiąc-Miśta \& Dziedzic, 2020). O acesso ao EPI tem sido um fator importante para a tomada de decisão quanto a continuar ou suspender a prática clínica durante a pandemia, 75,3\% dos entrevistados afirmaram não ter acesso suficiente (Tysiąc-Miśta \& Dziedzic, 2020). A junção destes fatores tem causado problemas financeiros para muitos consultórios na Polônia (Tysiąc-Miśta \& Dziedzic, 2020). A demanda reprimida de atendimentos odontológicos com agravamento dos quadros clínicos provavelmente acarretará na sobrecarga dos serviços públicos odontológicos no período póspandêmico (Cunha et al., 2021).

$\mathrm{Na}$ China, desde o começo da epidemia, houve resposta rápida das autoridades em relação às medidas protetivas de saúde pública, suspensão dos tratamentos eletivos, mantendo apenas hospitais e clínicas com serviços de urgência (Guo et al., 2020). Estudo identificou que a Covid-19 influenciou os padrões de utilização dos serviços odontológicos de urgência em Pequim, onde apesar da redução dos atendimentos eletivos em 30\%, houve também uma redução de $38 \%$ das urgências e mudança na distribuição dos problemas dentários (Guo et al., 2020). Na Itália, país que registrou em abril o terceiro maior número de casos Covid-19 e o segundo número oficial de óbitos do mundo, houve fechamento do serviço ou redução a procedimentos de urgência, 38,2\% antes e 61,8\% após o dia 10/03, data instituída pelo país para início das medidas restritivas (Consolo et al., 2020). Este estudo mostra a influência da pandemia nas práticas odontológicas uma vez que todos os dentistas relataram a alteração do uso de EPI, e 70\% dos proprietários de consultório afirmaram que o número médio de pacientes atendidos por dia era de 6 a 15 antes da pandemia, e passou para nenhum até 5 por semana em 90\% da amostra (Consolo et al., 2020). Esse fenômeno também foi observado na Polônia (Tysiąc-Miśta \& Dziedzic, 2020).

Ao final da escrita do estudo, em 02 de setembro de 2021, havia 20.830 .495 casos confirmados (acumulados) de Covid-19 no Brasil, com incidência de 9.912,3 casos/100.00 mil e mortalidade de 276,9/100.000 mil um total de 581.914 óbitos acumulados (Brasil, 2021). O país ocupa o terceiro lugar no mundo em relação ao número de infectados e o segundo lugar em número de óbitos. A vacinação iniciou no Brasil no mês de janeiro de 2021, e atualmente o país ocupa a terceira posição em relação ao número de pessoas vacinadas, apresentando 24,1\% da população imunizada (136.46 milhões) (Global Change Data Lab, 2021). O país segue atravessado por instabilidade no Estado e disputas de narrativas e posicionamentos entre o governo federal e governos estaduais. No cenário político, destaca-se também a instauração em abril de 2021 de uma Comissão Parlamentar de Inquérito (CPI), no Senado Federal, para investigar as ações e omissões do governo federal no enfrentamento da pandemia da Covid-19, bem como denúncias de corrupção envolvendo a compra de imunizantes (Senado Federal, 2021).

\section{Conclusão}

Observou-se que a decisão pela suspensão e retorno dos atendimentos odontológicos eletivos ou de rotina foi tomada pelas Secretarias Estaduais de Saúde, tendo emissão de publicação conjunta com os Conselhos Regionais de Odontologia (CRO) apenas em SC e no DF. Dos cinco casos analisados, o Ceará foi o único que explicitou a necessidade de retomada das atividades econômicas como critério para reabertura de serviços de saúde, incluindo os atendimentos eletivos odontológicos, e 
os CRO-CE e CRO-SP, as autarquias que pressionaram para essa reabertura.

A reabertura dos serviços odontológicos não obedeceu a nenhum critério de tendência da curva epidemiológica do número de casos e óbitos e da taxa de ocupação de leitos de UTI, e não há clareza dos documentos estaduais sobre mecanismos de controle, fiscalização e multa para serviços que descumpram as normas estabelecidas para o retorno do funcionamento, aspectos que comprometem a segurança de profissionais e usuários dos serviços, especialmente pelo uso de aerossóis. A participação e atuação dos CRO juntos às secretarias de saúde foram diferentes nos casos analisados. Estudos que aprofundem as motivações ao interior de cada estado, bem como as medidas de controle e regulação do setor saúde devem ser incentivados para melhor compreensão da resposta do Estado brasileiro frente à pandemia do COVID-19.

\section{Referências}

Almeida, I. F. B., Almeida, D. B. de, Santana, R. dos S., Oliveira, C. R. S. S., Pinto, N. F. de J., Farias, M. T. D., Bastos, A. O. da S., \& Santos, N. V. C. dos. (2020). Inserção de um dentista na comissão de saúde para enfrentamento da COVID-19 em um município brasileiro: perspectiva interdisciplinar. Research, Society and Development, 9(10), e8089109300. doi.org/10.33448/rsd-v9i10.9300

Amazonas. (2020a). Governo do Estado. Casos confirmados de Covid-19 no Amazonas chegam a 31.949. Retrieved Jul 05, 2020, from http://coronavirus.amazonas.am.gov.br/casos-confirmados-de-covid-19-no-amazonas-chegam-a-31-949/

Amazonas. (2020b). Governo do Estado. Secretaria de Estado de Saúde. Retrieved Ago 20, 2020, from http://www.saude.am.gov.br/painel/fvscovid.php

American Dental Association. (2020). ADA NEWS, 2020. ADA recommending dentists postpone elective procedures. Retrieved Jul 17, 2020, from https://www.ada.org/en/publications/ada-news/2020-archive/march/ada-recommending-dentists-postpone-elective-procedures

Atlas do Desenvolvimento Humano no Brasil. (2020). Perfil Estadual. Retrieved Jul 05, 2020, from http://www.atlasbrasil.org.br/2013/pt/perfil/

Barabari, P. \& Moharamzadeh, K. (2020). Novel Coronavirus (COVID-19) and Dentistry-A Comprehensive Review of Literature. Dent. J, 8, 53. doi.org/10.3390/dj8020053

Brasil (2020a). Ministério da Saúde. Nota Técnica nº 9/2020-CGSB/DESF/SAPS/MS. Atendimento Odontológico no SUS. Diário Oficial da União; Retrieved Jun 17, 2020, from https:/gestaodesaudepublica.com.br/download/nota-tecnica-no-9-2020-cgsb-desf-saps-ms-atendimento-odontologico/

Brasil. (2020b). Ministério da Saúde. Nota Técnica No 16/2020-CGSB/DESF/SAPS/MS. Covid-19 e atendimento odontológico no SUS. Retrieved Ago 19, 2020, from https://egestorab.saude.gov.br/image/?file=20200618_N_SEIMS-0014813177_ NT16COVID19ATENDIMENTOODONTOLOGICONOSUS_6282583358739045095.pdf

Brasil. (2020c). Ministério da Saúde. Nota Informativa No 1/2020-CGSB/DESF/SAPS/MS. Nota Informativa "Coleta de Swab por cirurgiões-dentistas no SUS”. Retrieved Jul, 2020, from https://website.cfo.org.br/wp-content/uploads/2020/06/SEI_MS-0014975480-Nota-Informativa.-VersA\%cc\%83\%c2\%a3ofinal-assinada.pdf

Brasil. (2021). Ministério da Saúde. Covid-19 Painel Coronavírus. Retrieved Set 02, 2021, from https://covid.saude.gov.br/

Carrer, F. C. A., Galante, M. L., Gabriel, M., Pischel, N., Giraldes, A. I., Neumann, A., Silva, D. P. \& Pucca Junior, G. A. (2020). A COVID-19 na América Latina e suas repercussões para odontologia. Rev Panam Salud Publica, 44, e66.

Cavalcanti, Y. C., Silva, R. O., Ferreira, L. F., Lucena, E. H. G., Souza, A. M. L. B., Cavalcante, D. F. B., Meneghim, M. C. \& Pereira, A. C. (2020). Economic impact of new biosafety recommendations for dental clinical practice during COVID-19 pandemic. Pesqui Bras Odontopediatria Clín Integr., 20(supp1), e0133. doi.org/10.1590/pboci.2020.143

Ceará. (2020a). Governo do Estado. Indicadores Coronavírus. Retrieved Ago $\quad$ 05, https://indicadores.integrasus.saude.ce.gov.br/indicadores/indicadores-coronavirus

Ceará. (2020b). Governo do Estado. Secretaria de Estado de Saúde. Retrieved Ago 20, 2020, from https://coronavirus.ceara.gov.br/

Conselho Federal de Odontologia. (2020). Estatísticas, Notícias e Publicações. Retrieved Ago 20, 2020, from www.cfo.org.br

Conselho Regional de Odontologia do Amazonas. (2020). Publicações sobre a Covid-19. Retrieved Ago 20, 2020, from http://croam.org.br/portal/

Conselho Regional de Odontologia do Ceará. (2020). Publicações sobre a Covid-19. Retrieved Ago 20, 2020, from http://www.cro-ce.org.br/

Conselho Regional de Odontologia do Distrito Federal. (2020). Publicações sobre a Covid-19. Retrieved Ago 20, 2020, from https://www.cro-df.org.br/

Conselho Regional de Odontologia de Santa Catarina. (2020). Publicações sobre a Covid-19. Retrieved Ago 20, 2020, from https://www.crosc.org.br/

Conselho Regional de Odontologia de São Paulo (2020). Publicações sobre a Covid-19. http://www.crosp.org.br/

Consolo, U., Bellini, P., Bencivenni, D., Iani, C. \& Checchi, V. (2020). Epidemiological Aspects and Psychological Reactions to COVID-19 of Dental Practitioners in the Northern Italy Districts of Modena and Reggio Emilia. Int J Environ Res Public Health, 17 (10), 3459. doi:10.3390/ijerph17103459 
Cunha, A. R., Velasco, S. R. M., Hugo, F. N. \& Antunes, J. L. F. (2021). The impact of the COVID-19 pandemic on the provision of dental procedures performed by the Brazilian Unified Health System: a syndemic perspective. Rev Bras Epidemiol, 24, E210028. doi.org/10.1590/1980-549720210028

Distrito Federal. (2020). Governo do Distrito Federal. Secretaria de Saúde. Retrieved Ago 20, 2020, from http://www.coronavirus.df.gov.br/; http://www.saude.df.gov.br/; https://agenciabrasilia.df.gov.br/

Fundação de Vigilância em Saúde do Amazonas (FVS-AM). (2020). Diretrizes de flexibilização gradual dos setores de comércio e serviços. Retrieved Jul 09, 2020, from http://www.fvs.am.gov.br/media/publicacao/diretrizes-de-flexibilizacao-gradual-dos-setores-de-comercio-e-servicos-covid_R7P8wji.pdf

Global Change Data Lab (GCDL). (2021). Coronavirus (COVID-19) Vaccinations - Statistics and Research - Our World in Data. Retrieved Set 02, 2021, from https://ourworldindata.org/covid-vaccinations

Globo.com (2020). SC tem 72 novos leitos de UTI para Covid-19; estado tem mais de 130 novos casos em 1 dia. https://g1.globo.com/sc/santacatarina/noticia/2020/04/27/sc-tem-72-novos-leitos-de-uti-em-5-municipios-estado-tem-mais-de-130-novos-casos-em-1-dia.ghtml

Grossman, S., Sandhu, P., Sproat, C. \& Patel, V. (2020). Provision of dental services at a single institution in the UK's epicentre during the COVID-19 pandemic. Br Dent J, 228, 964-970. doi.org/10.1038/s41415-020-1716-2

Guo, H., Zhou, Y., Liu, X. \& Tan, J. (2020). The impact of the COVID-19 epidemic on the utilization of emergency dental services. Journal of Dental Sciences. doi.org/10.1016/j.jds.2020.02.002

Instituto Brasileiro de Geografia e Estatística. (2019). Pesquisa Nacional por Amostra de Domicílios Contínua (PNAD). 2018 e 2019.

Instituto Brasileiro de Geografia e Estatística. (2020). Cidades e Estados. Retrieved Jul 05, 2020, from https://www.ibge.gov.br/cidades-e-estados

Lana, R., Coelho, F., Gomes, M., Cruz, O., Bastos, L., Villela, D. \& Codeço, C. (2020). Emergência do novo coronavírus (SARS-CoV-2) e o papel de uma vigilância nacional em saúde oportuna e efetiva. Cadernos de Saúde Pública, 36 (3), e00019620. doi.org/10.1590/0102-311x00019620

Lucena, E. H. G., Freire, A. R., Araújo, E. C. F. A., Lira, G. N. W., Brito, A. C. M., Padilha W. W. N. \& Cavalcanti, Y. W. (2020) Acesso em saúde bucal na atenção básica antes e após o início da pandemia de COVID19 no Brasil. Revista Pesquisa Brasileira em Odontopediatria e Clínica Integrada. doi.org/10.1590/SciELOPreprints.819

Marques, N. P., Silveira, D. M. M. L., Martelli, P. J. D. L., Martelli, D. R. B., Lucena, E. H. G. \& Martelli-Júnior, H. (2020). Brazilian Oral Medicine and public health system: The enormous impact of the COVID-19 Era. Oral Dis., O0, 1-2. doi.org/10.1111/odi.13677

Nunes, L., Ongaratto, A., Dionísio, D., Gonçalves, E.\& Barbosa, W. (2020). Os desafios da prática odontológica em tempos de pandemia. Revista Interface Integrando Fonoaudiologia e Odontologia, 1 (1), http://www.revistas.uniflu.edu.br:8088/seer/ojs3.0.2/index.php/interface/article/view/248/151

Organização Mundial da Saúde (OMS) \& Organização Pan-americana da Saúde (OPAS) (2020). Folha informativa - COVID-19 Principais informações. Retrieved Mai 10, 2020, from https://www.paho.org/pt/covid19.

Pereira, A. M. M. (2020). Estratégias de enfrentamento da pandemia pela Covid-19 no contexto internacional: reflexões para a ação. Nota Técnica. Observatório Fiocruz-Covid-19. Rio de Janeiro: Fiocruz. https://www.arca.fiocruz.br/bitstream/icict/42558/2/Estrat\%c3\%a9giaEnfrentamentoCovid-19.pdf.

Santa Catarina. (2020). Governo do Estado. Secretaria de Estado da Saúde. Publicações sobre a Covid-19. Retrieved Ago 20, 2020, from https://www.coronavirus.sc.gov.br/

Santos-Lopez, M. \& Catrian-Fernandez, H. (2020). COVID-19 y el Nuevo Rol del Odontólogo en el Equipo de Salud. Int. J. Odontostomat., 14 (3), 296-298. dx.doi.org/10.4067/S0718-381X202000030029

São Paulo. (2020a). Governo do Estado. Situação Epidemiológica. Retrieved Ago 05, 2020, from http://www.saude.sp.gov.br/cve-centro-de-vigilanciaepidemiologica-prof.-alexandre-vranjac/areas-de-vigilancia/doencas-de-transmissao-respiratoria/coronavirus-covid-19/situacao-epidemiologica

São Paulo. (2020b). Governo do Estado de São Paulo. Secretaria da Saúde. Retrieved Ago 20, 2020, from https://www.saopaulo.sp.gov.br/coronavirus

Senado Federal. (2021). Atividade Legislativa Comissões - CPIPANDEMIA. Retrieved Set 02, 2021, from https://legis.senado.leg.br/comissoes/comissao?codcol=2441

Tysiąc-Miśta, M. \& Dziedzic, A. (2020). The Attitudes and Professional Approaches of Dental Practitioners during the COVID-19 Outbreak in Poland: A Cross-Sectional Survey. Int. J. Environ. Res. Public Health, 17, 4703. doi: 10.3390/ijerph17134703.

Wikipédia (2017). Lista de unidades federativas do Brasil por IDH. Retrieved Jul 05, 2020, from https://pt.wikipedia.org/wiki/Lista_de_unidades_federativas_do_Brasil_por_IDH\#cite_note-PNUD_2017-2 